\title{
A Trema micrantha (L) Blume como matéria-prima para a produção de celulose
}

\author{
ELoy Barbosa Penna Ribeiro \\ Claudio Nazareno Reis Luz \\ Instituto Nacional de Pesquisas \\ da Amazônia
}

\section{SINOPSE}

São fornecidos dados sobre o estudo da Trems micrantha (L) Blume [Trema miocrantha, 1852], do ponto de vista de celulose e papel: obtenção de pasta química pelo processo Sulfato modalidade Soda-Enxofre; ensaios de alvejamento pelos processos CEHH, CED, CEDPD; resultados dos testes físico-mecânicos; análises químicas de madeira e estudo micrométrico. Essa essência é apresentada como uma espécie equivalente ou melhor que o Eucalyptus saligna para a obtençăo de pasta química

\section{INTRODUÇÃo}

Um dos objetivos da Secção de Celulose e Papel do INPA é a pesquisa de essências papeleiras de reflorestamento.

Essas essências de reflorestamento são escolhidas segundo vários critérios. Pode ser, por exemplo, uma madeira que apresen tou resultados excelentes quando do estudo individual de todas as espécies arbóreas de uma zona florestal. Outro critério seria o crescimento extraordinariamente rápido de uma árvore, que justificasse o seu estudo como essência de reflorestamento.

A Trema micrantha, entretanto, faz parte de um grupo de quatro ou cinco espécies de fácil proliferação em matas secundárias ou de capoeira, e para as quais se pode preconizar uma silvicultura com a regeneração natura! incentivada apenas, dada a abundância e mesmo agressiva proliferação dessas essências.

É conveniente ressaltar nesta Introdução, ser a pretenção deste trabalho apresentar a Trema micrantha tão somente quanto as suas possibilidades como matéria-prima para a produção de celulose. Árvore de distribuiçãc geográfica tão vasta, quanto antiga é a sua classificação taxonômica, ela por sí só nãu poderia ser apresentada nem como novidade nem como exclusividade amazônicas. No entanto, é possível que na Amazônia tenha a Trema micrantha um crescimento e uma ocorrência superiores a qualquer outra regiăo. Aliás, são justamente estas indagações, somadas às excelentes qualidades papeleiras, os motivos que elegem a Trema micrantha como essência papeleira de reflorestamento. Ademais, poderá a Secção de Celulose e $\mathrm{Pa}$. pel vir a realizar futuramente o estudo da 7 rema micrantha em mistura com as espécies de maior ocorrência e fácil proliferação em matas secundárias ou capoeiras.

DADOS BOTÂNICOS GERAIS E CARACTERISTICAS ANATÔMICAS DA TREMA MICRANTHA

[ABCP, 1968; Little et alii, 1967; Nevhing, 1960; Record, 1949]

Nome cientifico: Trema micrantha (L) Blume.

Familia: Ulmaceae.

Descrição botânica : Árvore monóica até $20 \mathrm{~m}$ de altura e $30 \mathrm{~cm}$ de diâmetro de fuste com os ramos pilosos e flexuosos. Folhas curtamente peceoladas, lanceoladas ou ovado-elipticas, atenuadas no ápice, serreadas, ásperas, 3-nervadas, até $10 \mathrm{~cm}$ de comprimento por 4 de largura. Flores polígamas, esverdeadobrancacentas, pequenas em cimeiras axilares Fruto (drupa) subgloboso, caruoso, $3-4 \mathrm{~mm}$ de comprimento e $3 \mathrm{~mm}$ de diâmetro, vermelho com uma semente negra.

Fenologia: Floresce e frutifica durante quase todo o ano.

Habitat: Espécie pioneira, ocorrendo em geral em capoeiras, terrenos abandonados e margens de estradas. 
Distribuição geogréfica: América tropical e subtropical, desde o centro e sul da Flórida até a Argentina.

Crescimento: Rápido e vida curta.

- Altura de uma árvore típica ... $7 \mathrm{~m}$

- Peso da madeira descascada fornecida pelo fuste de uma árvore, do tronco ao ápice :

Madeira a $45,62 \%$ de umi-

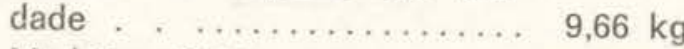

Madeira $100 \%$ seca ...... $\quad 5,25 \mathrm{~kg}$

- Diâmetro :

Tronco . . ............. $9,06 \mathrm{~cm}$

D.A.P. . . . . . . . . $8,00 \mathrm{~cm}$

4́pice . . ............. 5,56 cm

- Densidade :

Madeira $100 \%$ seca ....... 0,33

Madeira a $45,62 \%$ de umi-

dade

Nomes vulgares:

Brasil : Gurindiba, crindeúva, ceriúva, coatindiva, pau de pólvora.

Porto Rico: Palo de cabra, cabra.

Cuba: Guacimilla, guacimilla cimarrona guacimilla boba, capuli cimarrón.

República Dominicana: Memizo cimarrón. memiso de paloma, memiso.

México: Yaco de cuero, equipal.

América Central : Capulín.

Honduras: Capulín negro.

El Salvador: Capulín macho, capulín montes, capulincillo, churrusco.

Costa Rica : Juco, vara blanca.

Colômbia: Berraco, majagua colorada, raspador, venaco.

Venezuela : Mesaquilla.

Equador: Tortolero, muchichilón.

Peru: Aisegerina, atadijo, yana-caspi.

Argentina: Palo-pólvora, afta colorada.

Estados Unidos: Florida trema.

Jamaica: Jamaican nettle-tree.

Honduras Britânicas: White capulín, wild baycedar.

Haiti: Bois de soie.

\section{MATERIAL E MÉTODOS}

O material utilizado no presente estudo foi coletado nas matas de capoeira existentes na própria sede do INPA, situada em uma extensa área nas cercanias de Manaus. A madeira foi analisada e classificada pelos seto. res de Botânica e Anátomia de Madeiras.

O método empregado nas análises químicas da madeira, transformada em serragem, foram os elaborados pela A.B.C.P. (1968). A determinação do teor em celulose, entretanto, foi realizada pelo método KüschnerHoffer da AFNOR (1966).

A deslignificação da madeira foi efetuada pelo Processo Sulfato, modalidade Soda-Enxofre, por ser uma tendência geral cada vez maior a preferência por esse tratamento $e$ também por ser o mais indicado para a região do ponto de vista da aquisição dos reagentes químicos (Brasil, 1970).

Deve-se mencionar ainda que o Processo Soda-Enxofre é equivalente ao Processo Sulfato (Eisenber, 1971), de acordo com a seguinte equação :

$\mathrm{NaOH}$ a $22 \%+\mathrm{S}$ a $2,2 \%=\mathrm{NaOH}$ a $16,5 \%$ $+\mathrm{Na}_{2} \mathrm{~S}$ a $3,5 \%$.

Foram realizados cozimentos a dois níveis de $\mathrm{NaOH}$ e Enxofre: $22 \%$ e $20 \%, 2,2 \%$ e $2 \%$ de hidróxido de sódio e enxofre, respectivamente, em relação a madeira seca.

Os alvejamentos da celulose foram realizados pelos processos CEHH, CED e CEDPD. Nos alvejamentos com dióxido de cloro, empregou-se ainda uma quantidade de $\mathrm{H}_{2} \mathrm{O}_{2}$ como recurso para evitar o escurecimento da celulose e conseqüentemente melhor alvura.

Os testes físico-mecânicos foram realizados de acordo com as normas da A.B.C.F. Estes testes foram efetuados tanto para os papeis obtidos com a celulose crua, como sobre os fabricados com a pasta alvejada.

O refino foi efetuado em moinho Jokro.

\section{RESUltados}

I - RESUltados dos COZIMENTOS:

As condições e os resultados dos cozimentos são dados a seguir :

1. Cozimento (T.M. $18 \%$ )

Condiçōes :

$\mathrm{NaOH}$ em relação a madeira seca ................ $18 \%$ 
Enxofre em relação a madeira seca . . . . $1,8 \% \ldots \ldots \ldots \ldots \ldots$

Diluição . ................ 3,3:1

Temperatura de patamar .... $170^{\circ} \mathrm{C}$

Tempo para atingir a temperatura de patamar ......... $120 \mathrm{~min}$.

Tempo à temperatura de patamar .................

Resultados :

Rendimento total .......... 54,95\%

Rendimento depurado . . . . ... 53,98\%

Rejeitos (s/madeira seca) .... 0,97\%

$\mathrm{N} .{ }^{\circ}$ de $\mathrm{KMnO}_{4} \ldots \ldots \ldots \ldots \ldots .21,7$

Álcali residual ............. $0,956 \mathrm{~g} / 1$

2. Cozimento (T.M. 20\%)

Condiçōes :

$\mathrm{NaOH}$ em relação a madeira seca .............. 20\%

Enxofre em relaçäo a madeira seca . .............. $2 \%$

Diluição . ................ 3.3: 1

Temperatura de patamar .... $170^{\circ} \mathrm{C}$

Tempo para atingir a temperatura de patamar ...........

Tempo à temperatura de patamar

$120 \mathrm{~min}$.

$90 \mathrm{~min}$.

Resultados :

Rendimento total ...........

Rendimento depurado ........

Rejeitos (s/madeira seca) ..

$49,58 \%$

$49,57 \%$

$0,01 \%$

15,8

II - REsultados dos ensaios DE alvejamento:

São fornecidos a seguir as condiçōes e os resultados obtidos:

Alvejamento Clássico: Cloração - Extração Alcalina - Hipocloração - Hipocloraçãc

1." Fase :

Cloração direta.

2." Fase :

Extração alcalina.

Duração $60 \mathrm{~min}$.

Tentperatura ................. $60^{\circ} \mathrm{C}$,

Soda aplicada . ........... $4 \%$.

Consistência . .......... 5\%.

$\mathrm{pH}$ inicial . .............. 11,1 .

$\mathrm{pH}$ final . . . . $10,9 \ldots \ldots \ldots \ldots$.
3. Fase :

Hipocloração .

$\mathrm{NaOCl}$ aplicạda . .......... 1,81\%.

Soda aplicada . ........... $0,9 \%$.

Consistência . . .......... $5 \%$.

Duração ............... 360 min.

Temperatura . .......... 35 a $40^{\circ} \mathrm{C}$

$\mathrm{pH}$ inicial . ............. 10,3.

$\mathrm{pH}$ final . .............. 9,6 .

Cloro residual ............. $0 \%$.

Cloro consumido . ........ $1,81 \%$.

4. Fase :

Hipocloração.

$\mathrm{NaOCl}$ aplicado . .......... $0,25 \%$.

Soda aplicada $\ldots \ldots \ldots \ldots \ldots . \quad 0,35 \%$.

Consistência . . ............. $5 \%$.

Temperatura . . ........... 35 a $40^{\circ} \mathrm{C}$

$\mathrm{pH}$ inicial . ............... 9,9.

$\mathrm{pH}$ final $\ldots \ldots \ldots \ldots \ldots \ldots . . \ldots, 9,1$

Cloro residual . ............. $0 \%$.

Cloro consumido . ......... $0,25 \%$.

Alvejamento com Dióxido de Cloro:

Este alvejamento foi realizado em 5 fases.

1. Fase :

Cloração direta.

2. Fase :

Extração alcalina oxidante.

Soda aplicada . ............. $4 \%$

$\mathrm{H}_{2} \mathrm{O}_{2}$ aplicado ........................ $1 \%$

$\mathrm{pH}$ inicial . ................ 10,2 .

$\mathrm{pH}$ final .................. 9,8 .

Temperatura inicial ......... $60^{\circ} \mathrm{C}$.

temperatura final . .......... $60^{\circ} \mathrm{C}$.

Consistência . .............. $5 \%$.

Duração . . ............... 60 min.

$3^{\circ}$ Fase :

Dióxido de cloro.

$\mathrm{ClO}_{2}$ aplicado . ............ $1 \%$.

Cloro ativo aplicado ......... 2,63\%

Consistência . ........... 5\%.

Duração . . .............. 240 min.

Cloro ativo aplicado como água de cloro . . . . $0,526 \% \ldots \ldots \ldots . .$.

Cloro ativo aplicado como $\mathrm{NaClO}_{2} \quad 2,104 \%$.

$\mathrm{pH}$ inicial . .............. 4,2.

$\mathrm{pH}$ final $\ldots \ldots \ldots \ldots \ldots \ldots \ldots, 4,0$. 


\section{Fase :}

Peróxido de hidrogènio.

$\mathrm{H}_{2} \mathrm{O}_{2}$ aplicado . ........... $0,5 \%$.

$\mathrm{NaOH} . \ldots \ldots \ldots \ldots \ldots \ldots \ldots, 0,7 \%$.

Consistência ........... 5\%.

Duração . .............. 120 min.

$\mathrm{pH}$ final . ............... 10,6.

$\mathrm{pH}$ final ............... 10,6.

Temperatura ........... $60^{\circ} \mathrm{C}$.
$\mathrm{MgSO}_{4} \ldots \ldots \ldots \ldots \ldots \ldots \ldots, 0,1 \%$,

5. Fase :

Dióxide de cloro.

$\mathrm{ClO}_{2}$ aplicado . ........... $0,5 \%$

Cloro ativo aplicado ......... 1,315\%.

Consistência . .......... 5\% ,

Duraçäo . .............. 240 min.

Cloro ativo aplicado como água de cloro . ............. $0,263 \%$, Cloro ativo aplicado como $\mathrm{NaClO}_{2} \quad 1,052 \%$ $\mathrm{pH}$ inicial . ............. 5,2. $\mathrm{pH}$ final ............... 5,2. Temperatura . .......... $60^{\circ} \mathrm{C}$.

TABELA N.$^{\circ} 1$

$R E F I N O$

\begin{tabular}{|c|c|c|c|c|c|}
\hline \multirow{2}{*}{$\begin{array}{l}\text { Tempo de } \\
\text { refino } \\
\text { em min. }\end{array}$} & \multicolumn{5}{|c|}{ - $\mathrm{SR}$} \\
\hline & $\begin{array}{l}\text { PASTA } \\
\text { CRUA }\end{array}$ & CEHH & CED & CEDPD & $\begin{array}{l}\text { Eucalyptus } \\
\text { saligna } 5 \text { anos }\end{array}$ \\
\hline $0^{\prime}$ & 13 & 14 & 14 & 15 & 15 \\
\hline $15^{\prime}$ & 24 & 25 & 24 & 25 & 23 \\
\hline $30^{\prime}$ & 36 & 48 & 37 & 41 & 40 \\
\hline $45^{\prime}$ & 64 & 58 & 48 & 56 & 56 \\
\hline $60^{\prime}$ & - & - & - & 60 & - \\
\hline
\end{tabular}

TABELA N: 2

TESTES FISICO-MECÂINICOS E PHOTOVOLT

\begin{tabular}{|c|c|c|c|c|c|c|}
\hline & $\begin{array}{c}\text { AUTO } \\
\text { RUPTURA } \\
\text { m }\end{array}$ & $\begin{array}{l}\text { RASGO } \\
\mathrm{g} / 100 \mathrm{~g} / \mathrm{m}^{2}\end{array}$ & $\begin{array}{l}\text { ESTOURO } \\
\mathrm{Kg} / \mathrm{cm}^{2} \\
\text { PARA } \\
100 \mathrm{~g} / \mathrm{m}^{2}\end{array}$ & $\begin{array}{l}\text { DOBRAS } \\
\text { DUPLAS }\end{array}$ & $\begin{array}{l}\text { ESPESSURA } \\
\text { micra }\end{array}$ & $\begin{array}{l}\text { PHOTOVOLT } \\
\text { DE PASTA }\end{array}$ \\
\hline Pasta Crua & 9.825 & 89 & 5,84 & 1.523 & 66 & 32 \\
\hline CEHH & 8.206 & 67 & 4,75 & 2.232 & 64 & 92 \\
\hline CED & 7.524 & 73 & 4,47 & 1,832 & 67 & 87 \\
\hline CEPDP & 7.630 & 66 & 4,02 & 956 & 69 & 90 \\
\hline $\begin{array}{l}\text { Eucalyptus } \\
\text { saligna }\end{array}$ & 9.357 & 81,6 & 6,46 & 2.440 & 77 & - \\
\hline
\end{tabular}
DOS TESTES FÍSICO-MECÂ. NICOS DOS PAPEIS OBTIDOS

Pode-se observar, desde já, pela comparação entre os graus Shopper-Riegler dessas pastas, que o refino de celulose da Trema micrantha não constitui problema e que não houve degradação significante da pasta após os alvejamentos.

Esses resultados podem ser vistos nas tabelas $n .^{\circ} 1$ e n. 2 .
III - AVAliaçÃo DO REFINO DE CELUTOSE E RESUUTADOS 


\section{IV - RESULTADO DAS ANÁLISES QUíMICAS}

Estes resultados podem ser vistos na tabela $n{ }^{\circ} 3$. Os valores elevados em celulose e hemiceluloses concordam com os rendimen. tos excelentes obtidos nos cozimentos pelo processo soda-enxofre. Observe-se ainda o baixo teor em lignina. Muito relevante é o elevado teor em pentosanas, proporcionalmente ao valor encontrado para o Eucalyptus saligna. Este póde ser um dos dados que possa explicar as boas características de resis. tência dos papeis obtidos com a celulose de 7 rema micrantha.

TABELA N. 3

ANALISES QUIMICAS DA MADEIRA

\begin{tabular}{|c|c|c|c|c|c|c|c|}
\hline MADEIRA & $\begin{array}{c}\text { EXTRAÇĀO } \\
\text { COM } \\
\text { ALCOOLL- } \\
\text { BENZOL } \\
\%\end{array}$ & $\begin{array}{c}\text { EXTRAÇĀO } \\
\text { COM } \\
\text { AGUA } \\
\text { QUENTE } \\
\% \\
\%\end{array}$ & $\begin{array}{c}\text { EXTRAÇĀO } \\
\text { COM } \\
\text { AGUA } \\
\text { FRIA } \\
\% / 2\end{array}$ & $\begin{array}{c}\text { EXTRAÇ̄̄o } \\
\text { COM } \\
\text { SODA } 1 \% \\
\%\end{array}$ & $\begin{array}{l}\text { LIGNINA } \\
\% /\end{array}$ & $\begin{array}{l}\text { PENTO. } \\
\text { SANAS } \\
\%\end{array}$ & $\begin{array}{l}\text { CELULOjE } \\
\% /\end{array}$ \\
\hline $\begin{array}{l}\text { Trema } \\
\text { micrantha }\end{array}$ & 3,97 & 4,42 & 2,68 & 20,62 & 15,30 & 20,42 & 53,06 \\
\hline $\begin{array}{l}\text { Eucalyptus } \\
\text { saligna }\end{array}$ & 0,98 & 1,56 & 0,85 & 12,6 & 24,3 & 18,9 & 50,8 \\
\hline
\end{tabular}

V - CARACTERÍSTICAS MILIMÉTRICAS

DAS FIBRAS DE TREMA MICRANTHA

Com um índice de Feltragem tão baixo, era de se esperar um resultado menos satisfatório para a resistência ao rasgo. Isto, entretanto, não ocorreu, pois esta característica ainda se apresentou na Trema micrantha supe- rior ao Eucalyptus saligna.

Por sua vez, o Coeficiente de Flexibilidade relativamente alto, não é suficiente para jus. tificar o alto valor apresentado para o com. primento de auto-ruptura.

Os resultados das mensurações micrométricas podem ser vistos na Tabela $n^{\circ} 4$.

TABELA N: 4

CARACTERISTICAS MILIMÉTRICAS DAS FIBRAS DE TREMA MICRANTHA

\begin{tabular}{|c|c|c|c|c|c|c|c|c|c|c|c|c|c|c|c|c|}
\hline & con & $\begin{array}{c}\text { C } \\
\text { PRIME } \\
\text { (micra) }\end{array}$ & & & $\begin{array}{c}L \\
\text { ARGUR } \\
\text { (micra) }\end{array}$ & & & $\begin{array}{c}1 \\
\text { LÚMEN } \\
\text { (micra) }\end{array}$ & & $\begin{array}{l}\text { DES } \\
\text { PAD } \\
\text { (mi }\end{array}$ & & $\begin{array}{r}9 \\
\text { COEF } \\
\text { VARI }\end{array}$ & DE & 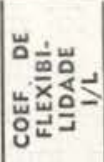 & 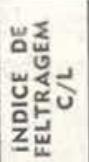 & 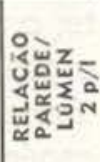 \\
\hline & Móx. & Mèd. & Min. & Máx. & Méd. & Min. & Máx. & Méd. & Min. & Comp. & Lorg. & Comp. & Lorg. & & & \\
\hline $\begin{array}{c}\text { Trema } \\
\text { micrantha }\end{array}$ & 1.050 & 727 & 430 & 31,4 & 22,3 & 18,8 & 18,8 & 11,0 & 6,3 & 130 & 10,8 & 1,78 & 0,49 & 49 & 33 & 1,03 \\
\hline $\begin{array}{c}\text { Eucalyptus } \\
\text { saligna }\end{array}$ & 1.260 & 858 & 450 & 15 & 20 & 25 & - & - & - & 142 & 27 & 16,5 & 13,5 & - & $\Delta 3$ & - \\
\hline
\end{tabular}




\section{CONCLUSÂO}

Pode-se concluir dos resultados apresentados, comparativamente aos do Eucalyptus saligna (Mazzei \& Overbeck, 1955), ser a Trema micrantha uma matéria-prima excelente para a obtenção de pasta química ou alvejada

Restam, contudo, certas indagações quanto à possibilidade real de um serviço floresta! com base nessa madeira, ser capaz de um fornecimento satisfatório a uma fábrica de grande porte.

Não se possuem dados concretos sobre o crescimento dessa espécie, mas sabe-se, pela observação empírica, que a Trema micrantha parece atingir sua altura máxima em tempo não superior a dois anos.

Igualmente não se possuem dados sobre o crescimento volumétrico dessa espécie nem do seu rendimento em madeira por hectares.

Considerando-se a assombrosa prolifera. çâo dessa árvore nas capoeiras, pcde-se preconizar um plantio à base de regeneração natural incentivada. Isso significa que uma mesma região estaria constantemente fornecendo madeira a partir das árvores que fossem atingindo a idade de corte, deixando intocadas aquelas em crescimento; ao mesmo tempo que se faria a eliminação das outras espécies que proliferam em capoeiras. Obterse-ia assim, um bosque homogêneo de Trema micrantha, com árvores em vários estágios de crescimento, fornecendo constantemente matéria-prima para a fábrica.

Todavia, deve-se mencionar aqui novamen te, o que já foi reiterado na Introdução, de que o presente trabalho sobre a Trema micrantha faz parte de um estudo das quatro ou cinco espécies de mais abundante proliferação em matas de capoeira.

\section{SUMMARY}

Data on the pulping study of Trema micrantha are given: Chemical cook by the sulphate process; bleaching trials by the classic and chlorine dioxide processes; strength caracteristics; chemical analysis and fiber measurements. Trema micrantha is concluded to be a good pulpwood, similar or even better than Eucalyptus saligna for making chemical pulp by the sulphate process

\section{BIBLIOGRAFIA CITADA}

ABCP (Associação Brasileira de Celulose e Papel) 1968 - Manual de Normas.

AFNOR (Associação Française de Normalisation)

1966 - Normes Françaises. Junho.

Brastl. Instituto Nacional de Pesquisas da AmazôniaINPA.

1970 - Programa de Pesquisas de Celulose e Papel para a Amazônia. Nogent-sur-Marne Centre Technique Forestier Tropical.

EISENBERG GROUP OF COMPANIES

1971 - Etude Papetier des bois de San Pedro Nogent-sur-Marne, Centre Technique Forestier Tropical, $126 \mathrm{p}$.

LITTLE, E. L. JR, et alii

1967 - Arboles comunes de Puerto Rico y les Islas Virgens. Puerto Rico, VPR. $827 \mathrm{p}$.

MAZZFI, F. M. \& OVERBECK, W.

1955 - Investigação da influencia da Idade nos característicos físicos e químicos do Lenho e das pastas celulósicas de Eucalyptus saligna. Publicação do Instituto de Pesquisas Tecnológicus, Sắo Paulo.

NEVHING, L. I. JR.

1960 - Flora of Panamá-Ulmaceae. An. Mis. Bot. Gard., $47(2): 105-113$

RECORD, S. J. \& HESS, R. W.

1949 - Timbers of the New World. New Haven Yale University. $640 \mathrm{p}$.

Trema micrantha Blume. Mus. Bot. Lugd. Bot. $1852-2(1-8): 63$, jan. 